\title{
Odontologia hospitalar: uma breve revisão
}

\author{
Hospital dentistry: a brief review \\ Odontología hospitalaria: una breve reseña
}

Maria Tays Pereira Santana ORCID: https://orcid.org/0000-0002-6503-7117 Universidade Federal de Campina Grande, Brasil E-mail: taayssantana@gmail.com

Viton Dyrk Guimarães Fernandes ORCID: https://orcid.org/0000-0003-4901-7391 Universidade Federal de Campina Grande, Brasil E-mail:vdyrk@yahoo.com

Josilanny Araújo de Souza Alencar ORCID: https://orcid.org/0000-0001-6539-0773 Universidade Federal de Campina Grande, Brasil E-mail: joylany@hotmail.com Filipe de Oliveira Lima ORCID: https://orcid.org/0000-0001-6652-3101 Universidade Federal de Campina Grande, Brasil E-mail: filipemaoliveirava@outlook.com

Amanda da Silva Araújo ORCID: https://orcid.org/0000-0003-1349-1833 Universidade Federal de Campina Grande, Brasil E-mail:mandiaraujo55@gmail.com

Quemuel Pereira da Silva

ORCID: https://orcid.org/0000-0002-0093-6322 Universidade Federal de Campina Grande, Brasil E-mail: quemuelpereira7@gmail.com

Paula Lima Nogueira

ORCID: https://orcid.org/0000-0001-7425-3201

Universidade Federal de Campina Grande, Brasil

E-mail: paulalimanogueira@hotmail.com

Maria Vitoria Oliveira Dantas

ORCID: https://orcid.org/0000-0001-7663-3793

Universidade Federal de Campina Grande, Brasil

E-mail: mvitoria.od@hotmail.com

Luanna Abílio Diniz Melquíades de Medeiros ORCID: https://orcid.org/0000-0002-1630-3968 Universidade Federal de Campina Grande, Brasil E-mail: luannaabiliod@gmail.com

Maria Angélica Sátyro Gomes Alves ORCID: https://orcid.org/0000-0003-3329-8360 Universidade Federal de Campina Grande, Brasil E-mail: angelicasatyro@ @otmail.com

Raline Mendonça dos Anjos ORCID: https://orcid.org/0000-0003-0751-7523 Universidade Federal de Campina Grande, Brasil

E-mail: raline.anjos@gmail.com

Elizandra Silva da Penha

ORCID: https://orcid.org/0000-0001-6264-5232 Universidade Federal de Campina Grande, Brasil

E-mail: elizandrapenha@ hotmail.com

Manuella Santos Carneiro Almeida ORCID: https://orcid.org/0000-0002-0288-6218 Universidade Federal de Campina Grande, Brasil

E-mail: manuellacarneiro@hotmail.com

Aleson Pereira de Sousa

ORCID: https://orcid.org/0000-0002-3430-477X Universidade Federal da Paraíba, Brasil

E-mail: aleson_155@hotmail.com

Abrahão Alves de Oliveira Filho ORCID: https://orcid.org/0000-0002-7466-9933 Universidade Federal de Campina Grande, Brasil E-mail: abrahao.farm@gmail.com 


\title{
Resumo
}

O presente estudo tem por objetivo realizar uma revisão de literatura do tipo narrativa sobre a atuação e as atribuições do cirurgião dentista no ambiente hospitalar. Trata-se de uma revisão narrativa de literatura, sendo analisados trabalhos publicados no SCIELO (Scientific Eletronic Library Online) e LILACS (Literatura Latino-Americana e do Caribe em Ciências da Saúde) com as palavras chaves "Hospital, Odontologia, Unidade de Terapia Intensiva". A inserção do cirurgião dentista na equipe multidisciplinar dos hospitais contribui para o tratamento dos pacientes hospitalizados, visto a existência da relação entre saúde oral e geral, assim as atribuições desses profissionais principalmente em Unidades de Terapia Intensiva corroboram para a manutenção da saúde bucal possibilitando qualidade de vida a partir de uma visão integral da saúde. Portanto, é de extrema importância a habilitação em odontologia hospitalar na formação do profissional cirurgião dentista.

Palavras-chave: Hospital; Odontologia; Unidade de Terapia Intensiva.

\begin{abstract}
This study aims to carry out a literature review of the narrative type on the performance and duties of the dental surgeon in the hospital environment. This is a narrative review of the literature, analyzing papers published in SCIELO (Scientific Electronic Library Online) and LILACS (Latin American and Caribbean Literature in Health Sciences) with the keywords "Hospital, Dentistry, Intensive Care Unit". The insertion of the dental surgeon in the team multidisciplinary approach to hospitals contributes to the treatment of hospitalized patients, given the existence of a relationship between oral and general health, so the duties of these professionals mainly in Intensive Care Units corroborate for the maintenance of oral health, enabling quality of life from an integral view Therefore, it is extremely important to qualify in hospital dentistry in the training of professional dental surgeons.
\end{abstract}

Keywords: Hospital; Dentistry; Intensive Care Unit.

\section{Resumen}

El presente estudio tiene como objetivo realizar una revisión de la literatura de tipo narrativo sobre el desempeño y las funciones del odontólogo en el ámbito hospitalario. Se trata de una revisión narrativa de la literatura, analizándose los trabajos publicados en SCIELO (Scientific Electronic Library Online) y LILACS (Literatura Latinoamericana y del Caribe en Ciencias de la Salud) con las palabras clave "Hospital, Odontología, Unidad de Cuidados Intensivos La inserción del cirujano dentista en el equipo multidisciplinario de los hospitales contribuye al tratamiento de los pacientes hospitalizados, dada la existencia de una relación entre la salud bucal y general, por lo que las funciones de estos profesionales principalmente en las Unidades de Cuidados Intensivos corroboran para el mantenimiento de la salud bucal, posibilitando calidad de vida desde una visión integral de la salud, por lo que es de suma importancia la titulación en odontología hospitalaria en la formación de cirujanos dentistas profesionales.

Palabras clave: Hospital; Odontología; Unidad de Cuidados Intensivos.

\section{Introdução}

A criação das profissões tem por finalidade atender as demandas originadas do próprio setor social, na odontologia em específico, a profissionalização ocorreu em função da necessidade de resolver a dor e o sofrimento decorrentes das doenças na cavidade bucal (Ring \& Lyda 1998), que aumentaram significadamente devido ao grande consumo de açúcar nos séculos XVIXVII (Carvalho, 2003; Lucietto, 2005).

Em 1879 ocorreu à criação dos cursos de cirurgia-dentária, anexos às Faculdades de Medicina, definindo que aos aprovados seria atribuído o título de "cirurgião-dentista". Em 1884, foi oficializado o ensino da Odontologia, contudo, apenas em 1933 os cursos tornaram-se autônomos das faculdades de medicina, dessa forma a criação de cursos no país impulsionou avanços na Odontologia brasileira, especialmente a partir dos anos 1970 (Carvalho, 1995; Fernandes, 2003).

A profissão odontológica possui regulamentação própria através da Lei 4.324/64, que instituiu os Conselhos Federal e Regionais de Odontologia (posteriormente regulamentada pelo Decreto 68.704/71) e da Lei 5.081/66, que regulamenta exercício da profissão no país (Brasil, 1964; Brasil, 1966).

O cirurgião dentista é um profissional da área da saúde responsável pelo tratamento de doenças associadas ao sistema estomatognático, que pode atuar em diferentes áreas como, por exemplo, Cirurgia e traumatologia bucomaxilofacial, Disfunção temporomandibular, Dentística, Harmonização facial e Odontologia hospitalar (Freitas, 2019).

A odontologia hospitalar é definida como um conjunto de ações preventivas, diagnósticas, terapêuticas e paliativas em saúde bucal, que são realizadas em instituições hospitalares no contexto de uma equipe multidisciplinar (Marín, Santos \& 
Bottan, 2017).

O objetivo do presente trabalho é desenvolver uma revisão de literatura narrativa sobre a atuação do cirurgião dentista no âmbito hospitalar.

\section{Metodologia}

Trata-se de uma revisão narrativa de literatura, que segundo Polit \& Beck (2011), consiste na apresentação de novas informações ao proporcionar conhecimentos atuais sobre o tema explorado ou enfatizar lacunas no corpo de pesquisas, e assim instigar pesquisadores a melhorar a base de dados científicos.

Foram analisados os trabalhos publicados nos bancos de dados, SCIELO (Scientific Eletronic Library Online) e LILACS (Literatura Latino-Americana e do Caribe em Ciências da Saúde), com as seguintes palavras chaves "Hospital, Odontologia, Unidade de Terapia Intensiva" abordando trabalhos publicados do ano de 1964 ao ano de 2020.

\section{Resultados e Discussão}

\section{Histórico}

A odontologia hospitalar no ocidente teve o seu avanço a partir da metade do século XIX, destacando-se os esforços dos doutores Simon Hullihen e James Garreston, mais tarde, a Associação Dental Americana e a classe médica apoiaram a inclusão do Cirurgião-Dentista em âmbito hospitalar (Meira, Oliveira \& Ramos, 2010).

Essa especialidade odontológica passou por processo significativo até ter seu exercício devidamente regularizado, a sua legitimidade ocorreu em 2004 a partir da criação da Associação Brasileira de Odontologia Hospitalar (ABRAOH) (Aranega, 2012).

No Brasil com a criação do Projeto de Lei (PL) no 2.776/2008, foi determinada a obrigatoriedade da presença de profissionais de odontologia nas UTIs e em hospitais públicos e privados, pois somente o Cirurgião Dentista, com os conhecimentos sobre a cavidade oral, suas características e microbiota, tem a habilitação própria e correta para atuar na área de promoção, educação e prevenção associada à saúde bucal de pacientes hospitalizados (Dantas et al., 2015).

Em fevereiro de 2010, foi elaborado pela ANVISA a Resolução da Diretoria Colegiada no 7 (RDC-07), que determina as condições básicas para funcionamento de UTIs, nela inclui a assistência odontológica a beira leito, deixando subentendido que o CD faz parte da equipe multidisciplinar, essa resolução entrou em vigor três anos após sua publicação, mas não especifica a carga horária mínima para este profissional (ANVISA, 2010).

A portaria 1032/2010 inclui serviços odontológicos na tabela de procedimentos ofertados pelo SUS a pacientes com necessidades especiais tratados em hospitais, além, de ressarcir aos profissionais que realizam esses procedimentos de alta complexidade, garante também que procedimentos antes feitos na atenção primária e secundária, poderão ser realizados na atenção terciária (Brasil, 2010).

Silva e Morais (2015) ressaltam que, somente no ano de 2005, em Barretos, cidade localizada no interior de São Paulo, é que a odontologia foi integrada à UTI, o que levou à execução de vários estudos, os quais demostram a necessidade do cirurgião dentista como membro da equipe multiprofissional destas unidades.

A capacitação do Cirurgião Dentista para que atue em ambiente hospitalar deve fazer parte do processo de sua formação na graduação e, atualmente, para o profissional já formado, é reconhecida pela resolução CFO-162/201518 (Brasil, 2015).

De acordo com o artigo 26 do Código de Ética Odontológico (capítulo X), que trata da Odontologia Hospitalar, compete ao cirurgião-dentista internar e assistir pacientes em hospitais públicos e privados, com e sem caráter filantrópico, respeitadas as normas técnico-administrativas das instituições. No artigo 27, dispõe-se que as atividades odontológicas 
exercidas em hospitais obedecerão às normas pertinentes, e o artigo 28 estabelece constituir infração ética fazer qualquer intervenção fora do âmbito legal da Odontologia (Brasil, 2012).

\section{Atuação do Cirurgião Dentista no Âmbito Hospitalar}

As áreas de atuação do cirurgião-dentista (CD) envolvem a esfera privada (clínicas e consultórios particulares), saúde suplementar (planos de saúde odontológicos), ensino e pesquisa (ligados a instituições de ensino) e a esfera pública (Sistema Único de Saúde- SUS), incluindo o ambiente hospitalar (Brasil, 2016).

Quanto ao ambiente hospitalar, a promoção de saúde bucal tem como finalidade oferecer assistência humanizada e integral ao paciente durante o período da internação, propiciando conhecimento e incentivando pacientes e acompanhantes a adquirirem bons hábitos de saúde oral (Brasil, 2016).

É necessário que o monitoramento dos diferentes órgãos e sistemas que não são tidos como as causas diretas das doenças de base do paciente não seja deixado de lado, essa atenção inviabiliza um maior comprometimento da saúde geral, auxiliando para um prognóstico mais favorável do caso (Coll et al., 2020).

As doenças crônicas caracterizam uma sociedade com prolongada permanência no hospital, intenso sofrimento, alta taxa de mortalidade e um alto consumo de recursos (Loss et al., 2017). Dentre essas doenças crônicas estão presente doenças respiratórias, condições coronárias avançadas, debilidade renal, doenças cardiovasculares, artrite e distúrbios emocionais ou psicológicos como ansiedade ou depressão (Coll et al., 2020).

De acordo com Silva e Morais (2015), os alvos para adequação bucal nos pacientes internados em UTIs são as cáries e restos radiculares, o biofilme da mucosa, doenças periodontais e lesões bucais, considerando-as nichos ecológicos e desencadeadores de mediadores inflamatórios que estão sendo colocados na corrente sanguínea e provocando alterações significativas no controle e expressão das doenças.

Além disso, os pacientes internados em UTI estão expostos a inúmeros outros fatores de risco, como a diminuição da limpeza natural da boca promovida pela mastigação de alimentos duros e fibrosos e a movimentação da língua e das bochechas durante a fala, acompanhados da redução do fluxo salivar causando xerostomia pela utilização de alguns medicamentos, que contribuem para o aumento do biofilme e favorecem a colonização oral por inúmeros microrganismos (Coll et al., 2020; Müller, 2015).

Segundo Liao, Tsai e Chou (2014), nos primeiros cinco dias de internação as bactérias que apresentam capacidade de proliferação da orofaringe são Streptococcus pneumoniae, Streptococcus aureus, Haemophilus in fluenzae e Enterobacter spp, entretanto, caso ocorra numa fase posterior de hospitalização, essa proliferação ocorre por bactérias gram-negativas, tais como a Pseudomonas aeruginosa, Acinetobacter spp e Staphylococcus aureus.

Uma higienização deficiente pode agravar uma condição oral pré-existente ou até mesmo colaborar para o desenvolvimento de infecções oportunistas causadas por vírus ou fungos, podendo levar a um comprometimento da resposta imune do paciente, dessa forma a participação do cirurgião-dentista, como profissional responsável pela manutenção da saúde bucal ou, como prestador de serviços realizados em nível hospitalar ou ambulatorial, tem por intuito colaborar, oferecer e agregar mais força ao que caracteriza a nova identidade do hospital - uma lacuna de integralidade da atenção e assistência (Marco et al.,2013; Miclos et al.,2013).

A assistência do paciente hospitalizado depende da interação do trabalho multiprofissional, onde a soma de pequenos cuidados parciais se complementa, quando se trata de uma Odontologia integrada a uma equipe multidisciplinar o indivíduo deve ser visto como um todo, e não apenas a região da cavidade bucal. (Dantas et al., 2015).

A atuação do cirurgião dentista no ambiente hospitalar é pautada essencialmente na perspectiva de integralidade (Saldanha et al., 2015). É importante que os cuidados sigam de maneira rigorosa os protocolos de descontaminação oral, além 
disso, o CD tem uma função de estimular a educação continuada da equipe de enfermagem bem como estar atento as necessidades apresentadas pelo paciente durante a evolução do quadro que poderá exigir cuidados mais complexos (Lobão et al., 2015).

De acordo com Silva e Morais (2015), cirurgião dentista desenvolve atividades diversificadas no âmbito hospitalar, compreendendo procedimentos como orientação de higiene, reembasamento de prótese, exodontias e tratamentos cirúrgicos em politraumatizados, podendo ainda diagnosticar e tratar patologias bucais e complicações decorrentes de tratamentos ou doenças sistêmicas complexas, bem como realizar biópsias e citologias esfoliativas, tanto no leito quanto em centros cirúrgicos.

Além do uso de clorexidina, a escovação e a aplicação tópica de flúor são primordiais para os pacientes internados na UTI, mostrando que os pacientes submetidos têm uma redução significativa na duração da ventilação mecânica e uma tendência para reduzir incidência de problemas sistêmicos como pneumonia nasocomical, endocardite bacteriana assim como a duração da internação na UTI (Lacerda et al.,2017).

Em pacientes sem consciência, consequentemente com sua atividade motora comprometida, utiliza-se um abridor de boca durante o atendimento, escovas dentais infantis e limpador de língua, além do uso uma gaze embebida em solução de clorexidina para limpar superfícies da mucosa e dentes, remover corpos estranhos e suç̧ão a vácuo para retirar excesso do antimicrobiano e saliva (Kim et al.,2014).

Os pacientes que serão submetidos a transplante de órgãos, como nos de transplante de medula óssea e renal, necessitam de um cuidado cauteloso do $\mathrm{CD}$, pois sua condição de saúde oral interfere no prognóstico do transplante, pois as infecções bucais podem levar a infecções sistêmicas que elevam os riscos de perda do enxerto e de morte, somente após a adequação do meio bucal esses pacientes podem ser liberados para a realização dos transplantes (Bezinelli, 2014).

Dessa forma, é de extrema importância e necessidade que o cirurgião dentista seja inserido na equipe multiprofissional dos hospitais, pois essa classe de profissionais exige baixo custo em sua implementação, possibilita uma alta resolutividade de agravos preexistentes e desenvolvem ações de promoção de saúde, concretizando o conceito de atenção integral à saúde (Santos et al., 2017).

\section{Conclusão}

Diante do exposto, pode-se concluir que a presença do cirurgião dentista na equipe multidisciplinar do ambiente hospitalar é de suma importância, visto que a aplicação dos conhecimentos que esse profissional apresenta no que se refere à manutenção da saúde oral contribui de maneira positiva no restabelecimento da saúde geral de pacientes hospitalizados, bem como redução de custos e tempo de internação, possibilitando assim uma integralidade de atenção e assistência à saúde.

\section{Referências}

Agência Nacional de Vigilância Sanitária (BRASIL). Resolução no. 7, de 24 de fevereiro de 2010. Dispõe sobre os requisitos mínimos para funcionamento de unidades de terapia intensiva e dá outras providências. Diário Oficial da União, 21 de agosto de 2006.

Aranega, A. M., Bassi, A. P. F., Ponzoni, D., Wayama, T. M., Esteves, J. C., \& Junior, I. R. G. (2012). Qual a importância da Odon tologia Hospitalar? Revista Brasileira de Odontologia, 69(1), 90.

Bezinelli, L. M. (2014). A Odontologia hospitalar nos hospitais públicos vinculados a secretaria do estado da saúde de São Paulo (Doctoral dissertation, Universidade de São Paulo).

Brasil. Conselho Federal de Odontologia. (2012). Código de ética Resolução Resolução CFO-118/2012.

Brasil. Conselho Federal de Odontologia. (2015). Resolução cfo-162/2015 Reconhece o exercício da Odontologia Hospitalar pelo cirurgião-dentista. Diário Oficial da União, Seção 1, DE 16/11/2015 Pg.167.

Brasil. Conselho Federal de Odontologia. (1964). Lei no 4.324/64, de14 de abril 1964, CFO. 
Brasil. Senado Federal. (1966). Lei no. 5.081, de 24 de agosto de 1966, Brasília (DF): Senado Federal.

Brasil. Ministério da saúde. (2010). Portaria no.1032, de 05 de maio de 2010. Inclui procedimento odontológico na tabela de procedimentos, medicamentos, órteses e próteses e materiais especiais do Sistema Único de Saúde - SUS, para atendimento às pessoas com necessidades especiais. Diário Oficial da União, Brasília, 5 de maio de 2010

Brasil. Ministério do Trabalho e Emprego. (2016). Descrição. Cirurgiões dentistas. Brasília (DF): MTE; 2016

Brasil. Projeto e Atividades legislativas. Senado Federal. (2016). Projetos e matérias legislativas.

Carvalho, A. C. P. D. (1995). Educaçäo e saúde em odontologia: ensino da prática e prática do ensino. In Educaçäo e saúde em odontologia: ensino da prática e prática do ensino (pp. 93-93).

Carvalho, C. L. (2003). Dentistas práticos no Brasil: história de exclusão e resistência na profissionalização da odontologia brasileira (Doctoral dissertation).

Coll, P. P., Lindsay, A., Meng, J., Gopalakrishna, A., Raghavendra, S., Bysani, P., \& O'Brien, D. (2020). The Prevention of Infections in Older Adults: Oral Health. Journal of the American Geriatrics Society, 68(2), 411-416.

Dantas, B. D. O., Araújo, I. A. D., Araújo, H. B. N. D., \& Araújo, E. C. (2016). Saúde bucal e cuidados na Unidade de Terapia Intensiva. Roplac, $28-32$.

Dantas, B. D. O., Araújo, I. A. D., Araújo, H. B. N. D., \& Araújo, E. C. (2016). Saúde bucal e cuidados na Unidade de Terapia Intensiva. Roplac, $28-32$.

Farias, K. R. A. (2019). Conhecimento da população a respeito da área de atuação do cirurgião-dentista e odontologia estética na cidade de maringá.

Fernandes Neto, A. J. (2002). A evolução dos cursos de Odontologia no Brasil. Rev. ABENO, 2(1), 55-56.

Iyda, M. (1998). Saúde bucal: uma prática social. In Ciências sociais e saúde bucal: questöes e perspectivas (pp. 127-139).

Kim, E. K., Jang, S. H., Choi, Y. H., Lee, K. S., Kim, Y. J., Kim, S. H., \& Lee, H. K. (2014). Effect of an oral hygienic care program for stroke patients in the intensive care unit. Yonsei medical journal, 55(1), 240-246.

Lacerda Vidal, C. F., de Lacerda Vidal, A. K., de Moura Monteiro, J. G., Cavalcanti, A., da Costa Henriques, A. P., Oliveira, M., \& Lacerda, H. R. (2017). Impact of oral hygiene involving toothbrushing versus chlorhexidine in the prevention of ventilator-associated pneumonia: a randomized study. $B M C$ infectious diseases, 17(1), 112.

Folha De Oliveira, I. V. A. N. I. L. D. E., \& Silva Wahuri, N. A. I. A. R. A. (2019). Atuação do cirurgião dentista em UTI: diminui o risco de pneumonia associada a ventilação mecânica.

Lobão, F. A. R., Duarte, M. V., Guerreiro, L., Palazzo, M., de Almeida, P., \& Vargas, G. (2016). O papel da Odontologia Inten siva. Academus Revista Científica da Saúde, 1(3).

Loss, S. H., Nunes, D. S. L., Franzosi, O. S., Salazar, G. S., Teixeira, C., \& Vieira, S. R. R. (2017). Chronic critical illness: are we saving patients or creating victims?. Revista Brasileira de terapia intensiva, 29(1), 87.

Lucietto, D. A. (2005). Percepções dos docentes e reflexões sobre o processo de formação dos estudantes de odontologia (Doctoral dissertation).

Marco, A. C., Cardoso, C. G., De Marco, F. V. C., Melo Filho, A. B. D., Santamaria, M. P., \& Jardini, M. A. N. (2013). Oral condition of critical patients and its correlation with ventilator-associated pneumonia: a pilot study. Revista de Odontologia da UNESP, 42(3), $182-187$.

Marín, C., Santos, M. H. N. D., \& Bottan, E. R. (2017). Dental surgeons' perceptions of hospital dentistry. Revista Brasileira de Odontologia, $74(1)$, 14-17.

Meira, S.C.R., Oliveira, C.A.S., \& Ramos, I.J.M. (2010) A importância da participação do cirurgião-dentista na equipe multiprofissional hospitalar. $9^{\circ}$ edição do prêmio SINOG de Odontologia. Curso de Odontologia do Centro Universitário Newton Paiva, Belo Horizonte/MG.

Miclos, P. V., Junior, M. F. S., Oliveira, C. M. S. C., \& Oliveira, M. A. D. (2013). Prática da promoção e educação em saúde bucal nos hospitais de grande porte da região metropolitana de Belo Horizonte, Minas Gerais. Arquivos em Odontologia, 49(2), 82-87.

Müller, F. (2015). Oral hygiene reduces the mortality from aspiration pneumonia in frail elders. Journal of dental research, 94(3_suppl), 14S-16S.

Polit, D. F., \& Beck, C. T. (2011). Fundamentos de pesquisa em enfermagem: avaliação de evidências para a prática da enfermagem. Artmed Editora.

Ring, M. E. (1998). História Ilustrada da Odontologia. Editora Manole.

Saldanha, K. F. D., da Costa, D. C., Peres, P. I., Oliveira, M. M., Masocatto, D. C., \& Jardim, E. C. G. (2015). A odontologia hospitalar: revisão. Archives Of Health Investigation, 4(1).

Santos, T. B., do Amaral, M. A., Peralta, N. G., \& Almeida, R. S. (2017). A inserção da Odontologia em Unidades de Terapia In tensiva. Journal of Health Sciences, 19(2), 83-88.

Silva, A., \& Morais, T. M. (2015). Fundamentos da Odontologia em ambiente hospitalar/UTI. Elsevier Brasil. 